\title{
Attosecond Time-Resolved Photoemission from Core and Valence States of Magnesium
}

\author{
S. Neppl, ${ }^{1,2, *}$ R. Ernstorfer, ${ }^{3,4}$ E. M. Bothschafter, ${ }^{3,2}$ A. L. Cavalieri, ${ }^{5}$ D. Menzel, ${ }^{1,4}$ J. V. Barth, ${ }^{1}$ F. Krausz, ${ }^{2}$ \\ R. Kienberger, ${ }^{3,2}$ and P. Feulner ${ }^{1}$ \\ ${ }^{1}$ Physikdepartment E20, Technische Universität München, 85747 Garching, Germany \\ ${ }^{2}$ Max-Planck-Institut für Quantenoptik, 85748 Garching, Germany \\ ${ }^{3}$ Physikdepartment E11, Technische Universität München, 85747 Garching, Germany \\ ${ }^{4}$ Fritz-Haber-Institut der Max-Planck-Gesellschaft, 14195 Berlin, Germany \\ ${ }^{5}$ Max-Planck Research Department for Structural Dynamics, University of Hamburg, \\ Center for Free Electron Laser Science, 22607 Hamburg, Germany
}

(Received 4 April 2012; published 22 August 2012)

\begin{abstract}
We report on laser-assisted attosecond photoemission from single-crystalline magnesium. In strong contrast to the previously investigated transition metal tungsten, photoelectron wave packets originating from the localized core level and delocalized valence-band states are launched simultaneously from the solid within the experimental uncertainty of 20 as. This phenomenon is shown to be compatible with a heuristic model based on free-particle-like propagation of the electron wave packets generated inside the crystal by the attosecond excitation pulse and their subsequent interaction with the assisting laser field at the metal-vacuum interface.
\end{abstract}

DOI: 10.1103/PhysRevLett.109.087401

PACS numbers: 78.47.J-, 68.35.Ja, 79.60.-i

Isolated attosecond ( 1 as $\left.=10^{-18} \mathrm{~s}\right)$ laser pulses in the extreme ultraviolet (XUV) spectral range allow timedomain access to fundamental electronic processes in atoms, molecules, and solids [1]. Applied to simple atomic systems, the combination of attosecond XUV pulses with inherently synchronized near-infrared (NIR) laser pulses has enabled the real-time observation of field-induced electron tunneling [2], the study of electron wave packet (EWP) motion [3], and the discovery of subtle time delays in the photoemission from single atoms [4,5] and diatomic molecules [6]. However, many fundamental and technologically pertinent subfemtosecond ( $\left.1 \mathrm{fs}=10^{-15} \mathrm{~s}\right)$ phenomena, like interfacial charge transfer [7,8], electronic screening [9], and interfacial electron transport [10], occur in condensed matter.

Up to now, time-domain attosecond spectroscopy of solids was demonstrated only in a single experiment on a tungsten (110) crystal, where a time delay of $\Delta \tau=$ $110 \pm 70$ as between photoelectrons emitted from the valence band (VB) and $4 f$ core level has been observed [10]. Such high time resolution can be achieved with the attosecond streaking technique, where one-photon photoemission (PE) is initiated by an isolated attosecond XUV pulse and the outgoing EWPs are streaked by a waveformcontrolled NIR laser field as the relative time delay $\tau$ between both laser fields is varied [11,12]. The resulting spectrograms $P(E, \tau)$ provide access to both the temporal structure and relative time delays in the release of EWPs, which are mapped onto corresponding relative shifts [13] between their NIR-modulated energy spectra $[4,10]$.

The feasibility of investigating photoemission in condensed matter with attosecond resolution, and the significant time delay observed in the first proof-of- principle experiment on $\mathrm{W}(110)$, prompted several theoretical studies of the phenomenon [15-21]. In the absence of a first-principles description, which is very challenging, one had to resort to simplified approaches that yielded quite contradictory predictions. In one approach [10,15], relying on the assumption of an abrupt screening of the incident NIR streaking field at the solid-vacuum interface, $\Delta \tau$ was explained in terms of transport effects within a simple semiclassical framework. According to these models, $\Delta \tau$ can be interpreted as the difference between the average travel times $\tau_{t}$ of the electrons emerging from weakly bound valence-band states and strongly bound core states to the crystal surface. This difference originates primarily from the different group velocities and escape depths of the two types of electrons. In contrast to this description, full quantum-mechanical calculations predict that the different localization of core and VB states alone is sufficient to cause substantial time delays in photoemission from solids (of the order of 100 as) - irrespective of any propagation effects $[16,17,19]$. Despite the use of significantly different approximations, both approaches reproduce the measured time delay for W(110) within the experimental error, which calls for additional experimental benchmarks from theoretically more accessible systems.

In this Letter, we present attosecond time-resolved PE from $\operatorname{Mg}(0001)$, which lends itself for this purpose for several reasons. First, the dielectric response of this free-electron metal implies-to a good approximationefficient screening of the NIR streaking field at the first atomic layer [22]. In this way, complications in the interpretation of attosecond streaking experiments related to a NIR penetration depth comparable to the escape depth of the emitted electrons are avoided [10,21]. Second, the VB 
states of this material are highly delocalized, in contrast to the $2 p$ states, providing an ideal test case for the quantummechanical models $[16,17,19]$ predicting sizable time delays arising solely from a different degree of initial-state localization. Finally, the simple free-electron-like character of the VB [23,24] offers the potential for analyzing the role of the nonlocal response of the surface to the NIR field in future studies, which was not easily possible in the case of tungsten because of its rather complex electronic structure [25].

A detailed description of the setup used for the timeresolved PE experiments has been published elsewhere [26]. To summarize, waveform-controlled, few-cycle $\sim 0.4 \mathrm{~mJ}$ NIR pulses with a carrier wavelength of $\sim 750 \mathrm{~nm}$ are produced by an amplified $3 \mathrm{kHz}$ Ti:sapphire laser system and focused into a neon-filled gas target to generate a collinear high-harmonic beam of coherent XUV radiation $[27,28]$. The NIR and XUV beam are spatially separated and the high-harmonic radiation is spectrally filtered by a multilayer mirror resulting in isolated attosecond pulses with a $\sim 4.2 \mathrm{eV}$ FWHM bandwidth centered near $\sim 118 \mathrm{eV}$, corresponding to a Fourier-limited pulse duration of $\sim 435$ as. The NIR and XUV pulses are focused under $15^{\circ}$ grazing incidence onto the crystal surface with the polarization tilted by $20^{\circ}$ relative to the surface normal.

Photoelectron spectra are recorded normal to the surface by a time-of-flight spectrometer with acceptance angles of $\sim 20^{\circ}$ and $\sim 15^{\circ}$ for valence and core-level emission, respectively. All PE data are corrected for this energydependent analyzer transmission. $\mathrm{Mg}$ films with final thicknesses of $>30$ layers were epitaxially grown on a clean W(110) substrate. The resultant metal films exhibit structural and electronic properties indistinguishable from single-crystalline $\mathrm{Mg}(0001)$ [24]. Both the $\mathrm{Mg}$ deposition and the subsequent time-resolved measurements were performed under ultrahigh vacuum conditions (base pressure $<10^{-10}$ mbar) with the sample at room temperature. In the following, all electron energies are given with respect to the Fermi level $E_{F}$.

A PE spectrum obtained with the sub-fs XUV pulses from $\operatorname{Mg}(0001)$ in the absence of the NIR field is depicted as a dotted line in Fig. 1(a), and is compared to a spectrum acquired with $120 \mathrm{eV}$ narrow-bandwidth synchrotron radiation (gray shaded area). For this excitation energy, the $\mathrm{PE}$ is dominated by the $2 p$ core-level electrons with binding energies of $\sim 50 \mathrm{eV}$. The comparably weak emission from the $3 s 3 p$-derived VB extends to $\sim 7 \mathrm{eV}$ below $E_{F}$ $[23,24]$. Residual features in the binding energy range of $\sim 7-15 \mathrm{eV}$ can be ascribed to plasmon losses associated with the primary VB transitions. The sharp emission line at $1.6 \mathrm{eV}$ stems from the well-known $\Gamma$-surface state, whose contribution to the total VB emission is negligible at this photon energy [23,24]. An approximation of the $\mathrm{Mg}(0001)$ PE data by a superposition of several Gaussian wave packets is indicated as a green solid line in Fig. 1(b).

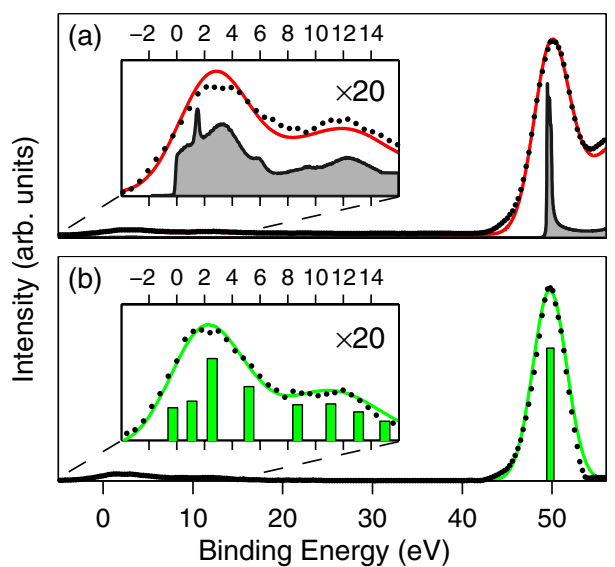

FIG. 1 (color online). Stationary photoemission from the $\mathrm{Mg}(0001)$ surface. (a) Comparison of PE spectra recorded with the $\sim 118 \mathrm{eV}$ sub-fs XUV pulses (dotted line) and $120 \mathrm{eV}$ narrowbandwidth synchrotron radiation (gray shaded area). A convolution of the synchrotron spectrum with the reflectivity response of the employed multilayer XUV mirror is depicted as a red solid line. (b) The PE spectrum after Shirley-background subtraction (dotted line) is compared to a PE spectrum reconstructed from a superposition of individual Gaussian time-domain wave packets (green solid line). The green vertical bars indicate the central energies and amplitudes of the considered transitions. Insets provide an enlarged view of the valence-band region.

For the attosecond streaking measurements, the XUVinduced PE is simultaneously dressed by a $10^{11} \mathrm{~W} / \mathrm{cm}^{2}$ NIR field. At this excitation level, above-threshold electrons originating from multiphoton absorption from the NIR field are effectively confined to kinetic energies $<40 \mathrm{eV}$. The background of inelastically scattered electrons in the spectral region of interest is removed based on the Shirley method [29]. The final spectrogram $P(E, \tau)$, shown in Fig. 2(a), is compiled from 40 individual PE spectra with the relative delay $\tau$ between the NIR and XUV pulses varied in increments of 150 as. Both VB and $2 p$ electron distributions clearly exhibit the characteristic oscillations with the NIR vector potential $A_{L}(t)$.

To determine the relative timing between the electrons released from the VB and the $2 p$ core states, we compare the center-of-energies (COEs) $E_{\mathrm{COE}}[30]$ of their streaked electron distributions as a function of $\tau$ (depicted as crosses in Fig. 3). Both $E_{\mathrm{COE}}(\tau)$ traces oscillate perfectly in phase without any discernible relative offset $\Delta \tau$ along the NIRXUV delay axis. This can be quantified by simultaneously fitting the VB and the $2 p$ traces to the same parametrized waveform

$$
A_{L}(\tau)=A_{0} e^{-2 \ln 2\left(\tau / \tau_{L}\right)^{2}} \sin \left(\omega_{L} \tau+\varphi_{0}\right)
$$

describing the NIR vector potential (solid lines in Fig. 3). Following this procedure, we do not only recover the pulse duration $\tau_{L}=5 \mathrm{fs}$, central photon energy $\omega_{L}=1.5 \mathrm{eV}$, and intensity $\left(\propto A_{0}^{2}\right)$ of the NIR pulses arriving at the 


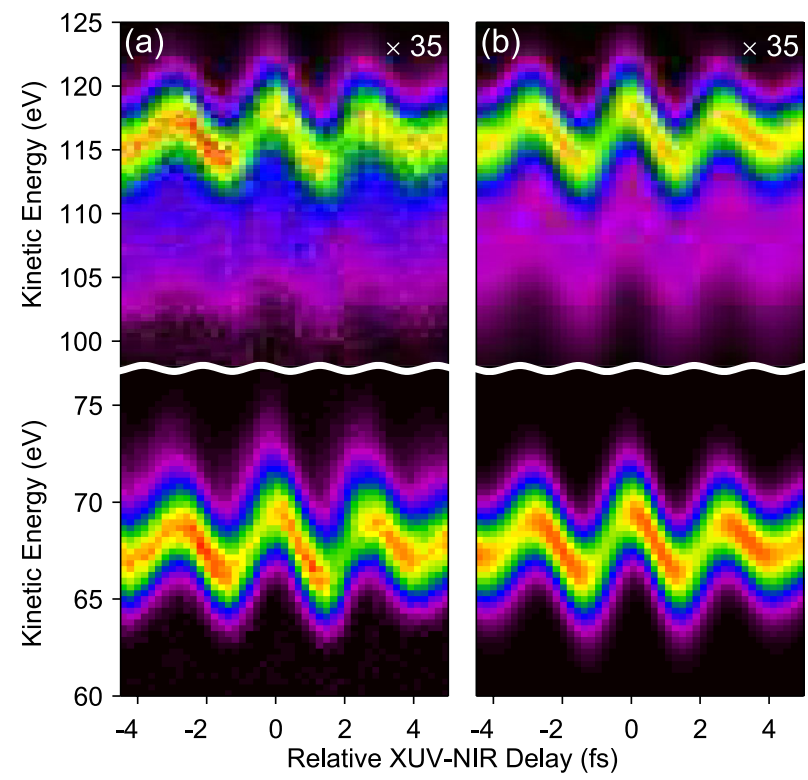

FIG. 2 (color online). Attosecond time-resolved PE spectra obtained from the clean $\mathrm{Mg}(0001)$ surface. Positive delays correspond to XUV arriving first. The false-color representation of the measured streaking spectrogram (a) is compared to a spectrogram (b) reconstructed by the quantum-mechanical fit procedure (see text for details).

sample, but also verify the absence of a relative time shift $\Delta \tau$, that is included as an additional fit parameter.

For a more in-depth analysis of the time-resolved PE data, we adapt a quantum-mechanical fitting scheme previously applied to retrieve the EWP properties from their streaked energy distributions [4,10]. In this scheme, a time-domain EWP $\psi_{i}(t)$ released from an initially bound

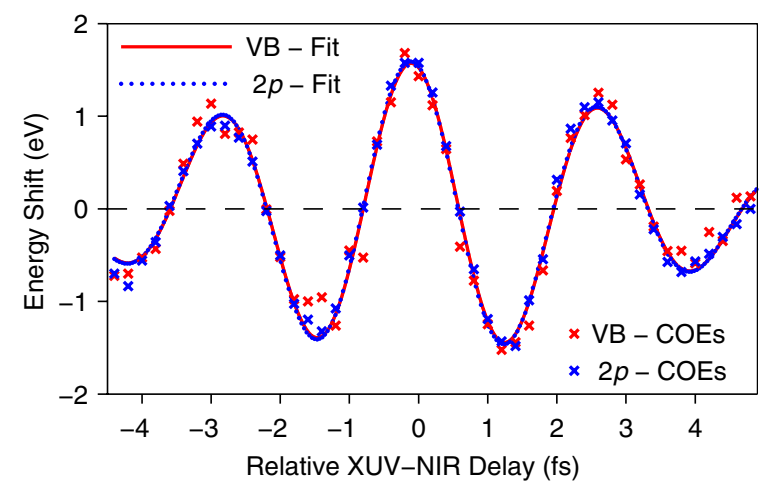

FIG. 3 (color online). Timing analysis of the VB and $2 p$ corelevel photoemission from $\mathrm{Mg}(0001)$. Calculated COEs for the $\mathrm{VB}$ and $2 p$ region of the spectrogram (depicted as crosses) are fitted to a pair of parametrized analytic functions describing the vector potential of the NIR streaking pulse [comp. Eq. (1)]. The absence of a relative shift between the fitted functions, shown as red solid line for the VB and blue dotted line for the $2 p$ emission, provides evidence for a synchronous release of these electrons from the metal surface. state $i$ into the laser-dressed continuum is represented by the complex function $\psi_{i}(t)=\psi_{0, i} \exp \left(-4 \ln 2\left(t / \tau_{e}\right)^{2}-\right.$ $\left.i E_{i} t+i \beta t^{2}\right)$, which is characterized by a Gaussian envelope with a FWHM $\tau_{e}$ and a linear chirp rate $\beta$. Its interaction with the laser field is taken into account within the strong field approximation, i.e., by setting $\psi_{i}(t) \rightarrow \psi_{i}(t) e^{-i \phi(t)}$, where $\phi(t)=\int_{t}^{\infty}\left[\sqrt{2 E_{i}} A_{L}\left(t^{\prime}\right)+\frac{1}{2} A_{L}\left(t^{\prime}\right)^{2}\right] d t^{\prime}$ denotes the Volkov phase and $A_{L}(t)$ takes the form as defined by Eq. (1). The corresponding energy spectra $P_{i}\left(E_{i}, \tau\right)$ as a function of the NIR-XUV delay $\tau$ are given by the squared modulus Fourier transform of $\psi_{i}(t)$. The final spectrogram is then calculated as the incoherent $\operatorname{sum} P=\sum_{i} P_{i}$ over all transitions contributing to the total PE current.

To reconstruct the time-resolved signal from $\operatorname{Mg}(0001)$, we model the spectrally broad VB emission phenomenologically as a superposition of eight individual transitions, while the electrons released from the $2 p$ core levels are represented by a single EWP. We also assume that all EWPs have the same duration $\tau_{e}$ and chirp $\beta$. Their central energies $E_{i}$ and relative emission intensities $\left|\psi_{i}(t)\right|^{2}$ are chosen to reproduce the stationary PE spectrum [see Fig. 1(b)] and kept constant in the subsequent fitting procedure optimizing $\tau_{e}, \beta, \tau_{L}, \omega_{L}, A_{0}$, and $\varphi_{0}$. In analogy to the analysis of the $E_{C O E}(\tau)$ traces, a possible time delay between the ensemble of VB transitions and the $2 p$ electrons is introduced as an additional optimization parameter in the fitting.

The best fit to the experimental data, shown in Fig. 2(b), is achieved with $\tau_{e}=480 \mathrm{as}, \beta=-2 \mathrm{fs}^{-2}$, and a vanishing time delay between the VB and $2 p$ wave packets. Averaging the corresponding time shifts extracted from 19 independently acquired streaking spectrograms still results in a negligible time delay of the $2 p$ electrons of only $\overline{\Delta \tau}=5 \pm 20$ as (standard deviation). Within the resolution of the experiment, this is fully consistent with the COE analysis shown in Fig. 3.

It is worth mentioning that the values for $\tau_{e}$ and $\beta$ are very similar to those derived from gas-phase streaking spectrograms recorded under identical experimental conditions. This suggests that the time evolution of the EWPs released from the metal surface is largely governed by the XUV excitation pulse, and experience only marginal temporal broadening and distortions within the solid. Such temporal effects might be expected since an EWP released from the surface contains contributions from different atomic layers of the crystal, which remain energetically unresolved but may give rise to a temporal smearing of the PE.

We begin our discussion of the quasisynchronous release of the photoelectrons from $\operatorname{Mg}(0001)$ by noting that the refractive index of this material is smaller than unity over the entire spectral range covered by the NIR pulses [31], implying total reflection at the metal surface for the $15^{\circ}$ grazing incidence used in the PE experiments. Therefore, thanks to the strongly damped evanescent wave within the 

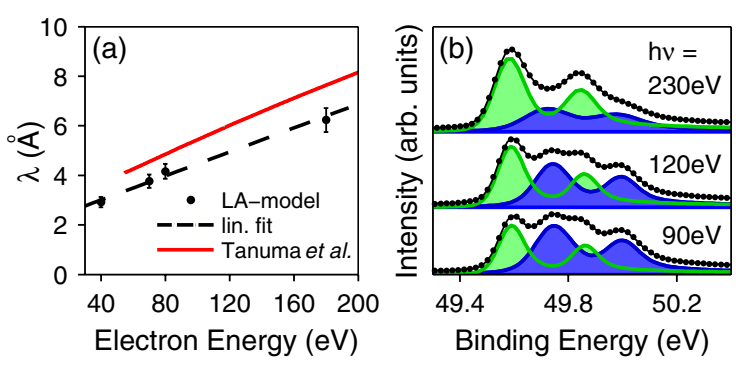

FIG. 4 (color online). Analysis of the spatial origin of the emitted photoelectrons. (a) Average escape depth $\lambda$ of electrons in $\mathrm{Mg}$ as a function of their kinetic energy according to Ref. [32] (red line). Values calculated from the surface-to-bulk ratios in the $2 p$ emission using a layer-attenuation (LA) model are depicted as dots along with a linear fit (dashed line). (b) High resolution PE spectra of the $2 p$ emission for different photon energies. The deconvolution in surface (light green shaded) and bulk contributions (dark blue shaded) is performed by fitting with two spin-orbit doublets of Doniach-Sunjić functions.

bulk, the assumption that the streaking field disappears inside the metal is even better justified than for W(110) [22]. Moreover, the final states of $\mathrm{Mg}(0001)$ with energies $30 \mathrm{eV}$ above $E_{F}$ are well described by a free-electron parabola [23]. It is therefore appropriate to estimate the average travel times $\tau_{t}$ of the VB and $2 p$ electrons towards the surface using their free-particle velocities $v_{i}=$ $\sqrt{2 E_{i} / m_{e}}$ and the free electron mass $m_{e}$. Taking the escape depths for $\sim 115 \mathrm{eV}\left(\lambda_{V B}=5.9 \AA\right)$ and $\sim 68 \mathrm{eV}$ electrons $\left(\lambda_{2 p}=4.8 \AA\right)$ in $\mathrm{Mg}$ from the most recent calculations [32] [red solid line in Fig. 4(a)], we find identical propagation times $\tau_{t}=92$ as for both types of electrons. This effect of cancellation between the electrons' velocities and escape depths $\lambda$, which results in a vanishing relative time delay in their release, is a general consequence of the monotonic increase of $\lambda$ in the energy interval of $60-120 \mathrm{eV}$.

Since theoretically derived values of $\lambda$ can be subject to considerable uncertainties at these low energies [32], an additional experimental verification of its energy dependence is needed. To this end, we analyze the branching $R$ of the $2 p$ emission into surface and bulk contributions in high-resolution synchrotron PE spectra [Fig. 4(b)]. In a layer attenuation (LA) model, $\lambda$ is related to the measured ratio $R$ by $\lambda=d / \ln (1+R)$, where $d=2.6 \AA$ is the interlayer spacing in $\operatorname{Mg}(0001)$ [33]. The derived values, fitted with a linear function (dashed line), are shown in Fig. 4(a) and confirm the general energy dependence of $\lambda$ in the relevant energy range. Although, the absolute experimental values are $\sim 1 \AA$ smaller than in Ref. [32], this only reduces the average propagation times for both types of electrons to $\tau_{t}=75$ as without, however, affecting the relative timing of their arrival at the surface.

Compared to the previously studied W(110) system, where the narrow $5 d$ bands imply at least partial localization of the $\mathrm{VB}$ states, $\mathrm{Mg}(0001)$ exhibits completely delocalized, free-electron-like states in the VB. As a consequence, recent quantum-mechanical models predicting substantial time delays $(\sim 100$ as) solely arising from differences in the degree of localization of the initial states $[16,17,19]$ should apply to this system even more than to tungsten. In sharp contrast, we find that-in spite of the strongly different degree of localization of the VB and $2 p$ states-electrons from these states reach the surface of the solid simultaneously, in agreement with a heuristic model accounting for propagation effects.

In conclusion, we applied attosecond time-resolved photoemission spectroscopy to the free-electron metal magnesium, which is more amenable to theoretical modeling compared to the previously investigated transition metal tungsten. In this material, valence-band and core-level photoelectrons are found to be emitted essentially simultaneously from the crystal surface. Our result indicates that transport-related contributions to attosecond time delays in solid-state photoemission can be satisfactorily explained by the knowledge of the electron wave packet's group velocities and their mean free path in the material-irrespective of the spatial localization of the initial states. This will facilitate the interpretation of time-resolved experiments on more complex phenomena like charge transfer or screening, where electron correlation is expected to further influence the release dynamics of the excited electrons on an attosecond time scale.

We thank E. Magerl, M. Stanislawski, M. Hofstetter, and N. Karpowicz for earlier contributions to the experimental setup and the data analysis, and C. Lemell, E. Krasovkii, A. Kazansky, and P. Echenique for discussions. S. N. and P.F. acknowledge financial support by the Munich Center of Advanced Photonics (Project B.1.4), and the HelmholtzZentrum Berlin; and thank the staff of BESSY-II for technical assistance during the synchrotron PE experiments. R. K. acknowledges funding from an ERC Starting Grant.

*To whom all correspondence should be addressed. s.neppl@mytum.de

[1] F. Krausz and M. Ivanov, Rev. Mod. Phys. 81, 163 (2009).

[2] M. Uiberacker et al., Nature (London) 446, 627 (2007).

[3] E. Goulielmakis et al., Nature (London) 466, 739 (2010).

[4] M. Schultze et al., Science 328, 1658 (2010).

[5] K. Klünder et al., Phys. Rev. Lett. 106, 143002 (2011).

[6] S. Haessler et al., Phys. Rev. A 80, 011404 (2009).

[7] C. Keller, M. Stichler, G. Comelli, F. Esch, S. Lizzit, W. Wurth, and D. Menzel, Phys. Rev. Lett. 80, 1774 (1998).

[8] A. Föhlisch, P. Feulner, F. Hennies, A. Fink, D. Menzel, D. Sanchez-Portal, P. M. Echenique, and W. Wurth, Nature (London) 436, 373 (2005).

[9] A. Borisov, D. Sánchez-Portal, R. D. Muiño, and P. M. Echenique, Chem. Phys. Lett. 387, 95 (2004). 
[10] A.L. Cavalieri et al., Nature (London) 449, 1029 (2007).

[11] J. Itatani, F. Quéré, G. L. Yudin, M. Y. Ivanov, F. Krausz, and P. B. Corkum, Phys. Rev. Lett. 88, 173903 (2002).

[12] R. Kienberger et al., Nature (London) 427, 817 (2004).

[13] A significant NIR-induced contribution to this shift can only be expected for electron energies $<50 \mathrm{eV}$ (see, e.g., Ref. [14]).

[14] C.-H. Zhang and U. Thumm, Phys. Rev. A 82, 043405 (2010).

[15] C. Lemell, B. Solleder, K. Tökési, and J. Burgdörfer, Phys. Rev. A 79, 062901 (2009).

[16] A. K. Kazansky and P. M. Echenique, Phys. Rev. Lett. 102, 177401 (2009).

[17] C.-H. Zhang and U. Thumm, Phys. Rev. Lett. 102, 123601 (2009).

[18] C.-H. Zhang and U. Thumm, Phys. Rev. A 84, 033401 (2011).

[19] C.-H. Zhang and U. Thumm, Phys. Rev. A 84, 065403 (2011).

[20] C.-H. Zhang and U. Thumm, Phys. Rev. A 84, 063403 (2011).

[21] E. E. Krasovskii, Phys. Rev. B 84, 195106 (2011).
[22] E.E. Krasovskii, V.M. Silkin, V.U. Nazarov, P. M. Echenique, and E. V. Chulkov, Phys. Rev. B 82, 125102 (2010).

[23] R.A. Bartynski, R.H. Gaylord, T. Gustafsson, and E. W. Plummer, Phys. Rev. B 33, 3644 (1986).

[24] F. Schiller, M. Heber, V. D. P. Servedio, and C. Laubschat, Phys. Rev. B 70, 125106 (2004).

[25] N.E. Christensen and B. Feuerbacher, Phys. Rev. B 10, 2349 (1974).

[26] E. Magerl et al., Rev. Sci. Instrum. 82, 063104 (2011).

[27] A. Baltuška et al., Nature (London) 421, 611 (2003).

[28] A. L. Cavalieri et al., New J. Phys. 9, 242 (2007).

[29] D. Shirley, Phys. Rev. 5, 4709 (1972).

[30] The COEs are defined by $E_{\mathrm{COE}}(\tau)=$ $\int_{E_{\min }}^{E_{\max }} P(E, \tau) E d E / \int_{E_{\min }}^{E_{\max }} P(E, \tau) d E-E_{0}$, and are evaluated between $E_{\min }=100(60)$ and $E_{\max }=125(75) \mathrm{eV}$ for the $\operatorname{VB}(2 p)$ electrons. $E_{0}$ denotes the $\mathrm{COE}$ far from temporal NIR-XUV overlap.

[31] E. Palik, Handbook of Optical Constants of Solids (Academic, New York, 1991).

[32] S. Tanuma, C. J. Powell, and D. R. Penn, Surf. Interface Anal. 43, 689 (2011).

[33] R. Kammerer, J. Barth, F. Gerken, C. Kunz, S. A. Flodstrøm, and L. I. Johansson, Phys. Rev. B 26, 3491 (1982). 